\title{
Hetonic quartets in a two-layer quasi-geostrophic flow: V-states and stability
}

\author{
J. N. Reinaud, ${ }^{1, a)}$ M. A. Sokolovskiy, ${ }^{2,3, b)}$ and X. Carton ${ }^{4, c)}$ \\ ${ }^{1}$ Mathematical Institute, University of St. Andrews, North Haugh, St. Andrews KY169SS, United Kingdom \\ ${ }^{2}$ Institute of Water Problems, Russian Academy of Science, 3 Gubkina Street, 119333 Moscow, Russia \\ ${ }^{3}$ Shirshov Institute of Oceanology, Russian Academy of Science, 36 Nahimovskiy Prospekt, \\ 117997 Moscow, Russia \\ ${ }^{4}$ Laboratoire d'Océanographie Physique et Spatiale, IUEM, UBO/UBL, Technopole Brest Iroise, \\ 29280 Plouzane, France
}

(Received 28 February 2018; accepted 25 April 2018; published online 11 May 2018)

\begin{abstract}
We investigate families of finite core vortex quartets in mutual equilibrium in a two-layer quasigeostrophic flow. The finite core solutions stem from known solutions for discrete (singular) vortex quartets. Two vortices lie in the top layer and two vortices lie in the bottom layer. Two vortices have a positive potential vorticity anomaly, while the two others have negative potential vorticity anomaly. The vortex configurations are therefore related to the baroclinic dipoles known in the literature as hetons. Two main branches of solutions exist depending on the arrangement of the vortices: the translating zigzag-shaped hetonic quartets and the rotating zigzag-shaped hetonic quartets. By addressing their linear stability, we show that while the rotating quartets can be unstable over a large range of the parameter space, most translating quartets are stable. This has implications on the longevity of such vortex equilibria in the oceans. Published by AIP Publishing. https://doi.org/10.1063/1.5027181
\end{abstract}

\section{INTRODUCTION}

The notion of heton was introduced by Hogg and Stommel ${ }^{1}$ to emphasise the ability of a discrete baroclinic vortex pair to transfer heat. The word "heton" is indeed derived from the word "heat." When both the geostrophic and hydrostatic approximations are valid, any vortex of the top (respectively, bottom) layer, which has negative (respectively, positive) intensity, induces a downward local distortion of the interface between the layers. Such a configuration is referred to as a "hot heton." When the sign of the vorticity of each vortex changes, the sign of the curvature of the interface changes as well, and the situation is referred to as a "cold heton."

Hetons exist and have been observed in the ocean: they are the association of a surface (or subsurface) vortex and of a deeper vortex of opposite polarity. The first mention of an observed heton dates from the early 1980s from Gulf Stream ring observations. ${ }^{2}$ Cold core rings detaching south of the Gulf Stream can pair with subsurface anticyclones containing Sargasso Sea water. More recently, hetons were observed in the Northeastern Atlantic Ocean, near the Iberian Peninsula. Indeed, when the outflow of Mediterranean water, which follows the Iberian Peninsula, becomes unstable, eddies detach from it as baroclinic dipoles (hetons). In this case, the anticyclone lies slightly deeper than the cyclone, in the heton. Such hetons can collide: then, two cyclones or two anticyclones can interact $;{ }^{3}$ for instance, the two anticyclones can merge, while the cyclones remain in their periphery or are ejected away. ${ }^{4}$

\footnotetext{
a)Electronic mail: jean.reinaud@st-andrews.ac.uk

b)Electronic mail: sokolovskiy@iwp.ru

c)Electronic mail: xavier.carton@univ-brest.fr
}

Such an interaction can create a new heton which can be asymmetric (for instance, the anticyclone is larger and stronger than the cyclone).

The equations of motion of a system of discrete vortices in a two-layer rotating fluid were first obtained by Gryanik ${ }^{5}$ and then successfully used in numerous studies. ${ }^{1,6-15}$

The notion of "hetonic quartet" was introduced in the literature ${ }^{16}$ for four discrete vortices lying in the same vertical plane, in a two-layer flow. Such a hetonic quartet moves at a constant translation velocity in a direction perpendicular to the plane containing the vortices. A structure consisting of four vortices (two hetons) belongs to the class of hetonic quartets only if the distances between the vortices satisfy a special condition, an analog of the dispersion relation in the wave theory. These quartets are referred to as zigzag-shaped translating hetonic quartets. ${ }^{15}$ Another form of coplanar configurations of four vortices in equilibrium in a two-layer fluid consists of vortex quartets in solid-state rotation at a constant angular velocity and is referred to as zigzag-shaped rotating hetonic quartets. ${ }^{15}$

In this paper, we extend these results to finite core zigzagshaped hetonic quartets. We first determine numerically equilibrium states (or V-states) for both rotating and translating vortex quartets. We next address their linear stability. For the sake of completeness, we also address the linear stability of the equivalent discrete quartets. Studying configurations of vortices in mutual equilibrium is essential as they are the only configurations which can persist, if stable, for long times. We show that in the range of parameters investigated, most of the translating quartets are stable, except when the two vortices, lying in different layers, nearer the centre of the structure are almost aligned vertically. The instability is associated with 
the displacement of the vortices and is captured in the linear stability analysis of both finite core vortices and discrete vortices. On the other hand, the rotating quartets are found unstable over a wider range of parameters. Again, the instability is related to the displacement of the vortices and is captured in the linear stability analysis of both finite core discrete quartets. Interestingly, in contrast to the translating quartets, the rotating quartets are linearly stable if the central vortices are nearly aligned. We finally investigate the nonlinear time evolution of the quartets on a few selected examples.

\section{PROBLEM SETUP}

\section{A. Mathematical and numerical models}

The oceans and the atmosphere are relatively shallow layers of stratified fluid where the typical horizontal scales are much larger than the typical height of the fluid. These environments can be conveniently represented by (shallow) horizontal layers of fluid of uniform density each in hydrostatic balance. A flow in such a multi-layer system is governed by the Saint-Venant or shallow water equations. ${ }^{17} \mathrm{~A}$ twolayer system can be seen as the simplest model to take into account the background stable density stratification at leading order. Large scale oceanic flows are also strongly influenced by the background planetary rotation. For simplicity, we assume a uniform background rotation quantified by the Coriolis frequency $f$.

We consider here a system of two layers of fluid of equal height at rest $h_{1}=h_{2}=h=H / 2$, where $H$ is the total fluid height. In all our expressions, the subscript 1 refers to a quantity defined in the top fluid layer, while the subscript 2 refers to a quantity defined in the bottom layer. We set $H=1$ without loss of generality. The horizontal coordinates $(x, y)$ are normalised by a characteristic length scale $L$. In practice, $L$ is set to the mean horizontal radius of the finite core vortices; hence, the finite core vortices have a non-dimensional radius $R=1$. We assume that the difference between the density $\rho_{1}$ of layer 1 and the density $\rho_{2}$ of layer $2, \rho_{2}-\rho_{1}=\Delta \rho \ll \rho_{1}$, $\rho_{2}$. We assume that the relative vertical vorticity is small compared to the Coriolis frequency and that the height perturbation at the interface between the two layers is small compared to the mean height of the layers. Under these assumptions, the dynamics of the flow is well captured by the shallow-water quasi-geostrophic model. The flow can be fully described in terms of potential vorticity (PV). ${ }^{17}$ The quasi-geostrophic PV anomaly $q_{j}$ in layer $j$ is defined by

$$
q_{j}=\Delta \psi_{j}+\frac{L}{L_{D}^{2}}\left(\psi_{3-j}-\psi_{j}\right), \quad j=1,2,
$$

where $\psi_{j}$ is the streamfunction of layer $j$ and $L_{D}=\sqrt{g^{\prime} h} / f$ is the Rossby deformation radius. Here, $g^{\prime}=g \Delta \rho / \rho_{1}$ is the reduced gravity, with $g$ being the gravitational acceleration. Equation (1) shows that $\mathrm{PV}$ in layer $j$ depends on the flow in the other layer $3-j$. The flows in layers 1 and 2 are thus coupled. The velocity field $\boldsymbol{u}_{j}=\left(u_{j}, v_{j}\right)$ in layer $j$ derives from the streamfunction $\psi_{j}$ as

$$
u_{j}=-\frac{\partial \psi_{j}}{\partial y}, \quad v_{j}=\frac{\partial \psi_{j}}{\partial x}
$$

We consider an adiabatic and inviscid flow. In this case, $\mathrm{PV}$ is materially conserved,

$$
\frac{D q_{j}}{D t}=0 .
$$

The problem is conveniently decoupled by defining the barotropic streamfunction $\psi_{b}$ associated with the baroclinic PV $q_{b}$ and the baroclinic streamfunction $\psi_{b}$ associated with the barotropic PV $q_{c}$,

$$
\psi_{b}=\frac{\psi_{1}+\psi_{2}}{2}, \quad \psi_{c}=\frac{\psi_{1}-\psi_{2}}{2}
$$

with

$$
q_{b}=\Delta \psi_{b}, \quad q_{c}=\Delta \psi_{c}-\gamma^{2} \psi_{c},
$$

where $\gamma=\sqrt{2} L / L_{D}$. These equations can be formally inverted using the appropriate Green's functions, namely,

$G_{b}\left(x-x^{\prime}\right)=\frac{1}{2 \pi} \ln \left|x-x^{\prime}\right|, \quad G_{c}\left(x-x^{\prime}\right)=-\frac{1}{2 \pi} K_{0}\left(\gamma\left|x-x^{\prime}\right|\right)$,

where the subscript $b$ refers to the barotropic mode and the subscript $c$ refers to the baroclinic one, and $\mathrm{K}_{0}$ is the zeroth order modified Bessel function of the second kind. The flow in the layer $j$ is simply recovered by projecting the barotropic and baroclinic modes back into the layers, $\psi_{1}=\psi_{b}+\psi_{c}$ and $\psi_{2}=\psi_{b}-\psi_{c}$. By construction, Green's functions provide the solution for point vortices. Differentiating Green's functions provides the kernels to evaluate the velocity field, while the second order derivatives provide the velocity gradients which are used for the linear stability of the point vortex quartets. For finite core vortices containing uniform PV defined by their bounding contours, inverting (6) results in evaluating surface integrals. These surface integrals are transformed into contour integrals using Green's theorem. ${ }^{18}$ Numerical simulations of the nonlinear time evolution of the finite core quartets are performed using contour surgery, ${ }^{19}$ which is an extension of the method contour dynamics ${ }^{20,21}$ controlling the complexity of the calculation by performing topological "surgery" on the vortex bounding contours.

We determine numerically equilibrium solutions for finite core hetonic quartets. The numerical methods are iterative and make the vortex bounding contours converge to streamlines, in the relevant rotating/translating reference frame. We have used two different methods to evaluate and determine the equilibrium states. The first one uses a partial linearisation of the streamfunction and has been successfully applied in many previous studies. ${ }^{18,22-27}$ For the translating hetonic quartets, the convergence of the method proved slow in practice. To remedy this problem, a new numerical tool was developed, in which the streamfunction is fully linearised. The method is an extension of an existing method for two dimensional and single-layer shallow water flows ${ }^{28-31}$ to the two-layer problem. Results stemming from the two methods were compared, when available, and the results are identical to the accuracy of the calculations. The linear stability analysis of the finite-core configuration is done by analyzing azimuthal modes of deformation of the vortices' bounding contours. ${ }^{18}$ The numerical method also allows analyzing the global displacement of the contours and hence the mode which physically represents a shift in the vortices relative positions. 


\section{B. Position of the discrete vortices at equilibrium}

Figure 1 provides a schematic representation of the four vortex configurations in a two-layer fluid for both (a) the rotating zigzag-shaped hetonic and (b) the translating zigzagshaped hetonic quartets, for the finite-core (not discrete) vortices. We use the same notation for the distances separating both discrete and finite-core vortices. We can see that if we mentally draw a line connecting the centres of the vortices then we obtain a zigzag, hence the name given to the quartets. In both cases, the horizontal distance between the inner vortices is denoted $d$, while the distance between the outer vortices is $b$. For convenience, we define the non-dimensional distances $D=\gamma d$ and $B=\gamma b$. The dispersion equations $F(D, B)=0$ for the discrete stationary solutions and the expressions for the corresponding translational $V$ and angular $\omega$ velocities are ${ }^{15}$

$$
\frac{(D-B)^{2}}{2 D B(D+B)}=2 \mathrm{~K}_{1}(B+D)+\mathrm{K}_{1}(2 D)+\mathrm{K}_{1}(2 B)
$$

and

$V=\frac{\kappa}{2 \pi}\left[\frac{D^{2}+4 D B-B^{2}}{2 D\left(B^{2}-D^{2}\right)}+\mathrm{K}_{1}(D+B)-\mathrm{K}_{1}(B-D)+\mathrm{K}_{1}(2 D)\right]$

for the translating zigzag-shaped hetonic quartet and

$$
\begin{aligned}
\frac{B^{2}+D^{2}}{2 D B}= & (B+D) \mathrm{K}_{1}(B+D)+(B-D) \mathrm{K}_{1}(B-D) \\
& +D \mathrm{~K}_{1}(2 B)+B \mathrm{~K}_{1}(2 D)
\end{aligned}
$$

and

$\omega=\frac{\kappa \gamma^{2}}{4 \pi D}\left[\frac{B^{2}+3 D^{2}}{2 D\left(B^{2}-D^{2}\right)}-\mathrm{K}_{1}(B+D)-\mathrm{K}_{1}(2 D)-\mathrm{K}_{1}(B-D)\right]$

for the rotating zigzag-shaped hetonic quartet. It should be noted that the dispersion relations collapse to single curves for
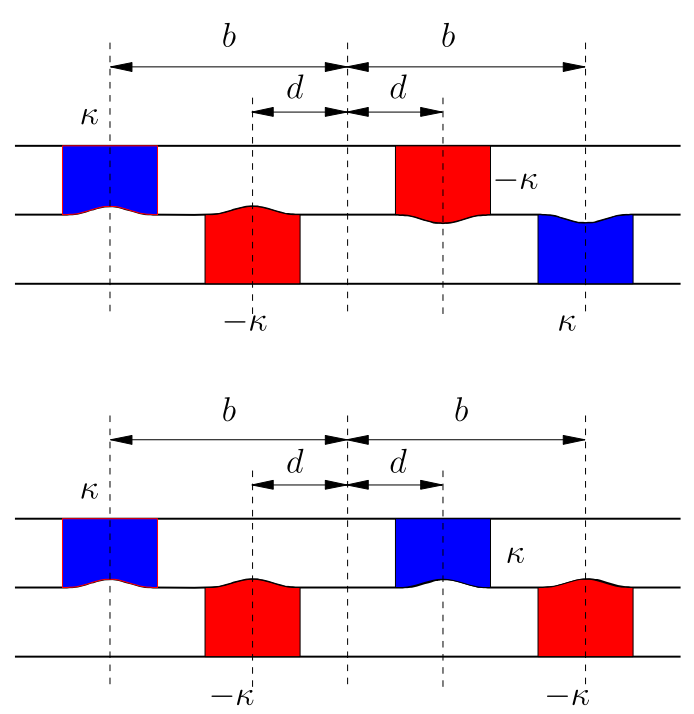

FIG. 1. General geometry of the hetonic quartets. The panels show a vertical cross section of coplanar finite core vortices: (a) rotating zigzag-shaped hetonic quartets (cold and hot hetons), (b) translating zigzag-shaped hetonic quartets (two cold hetons). Cyclones are in blue and anticyclones are in red. $\kappa>0$ is the surface integrated PV. variables $B$ and $D$. This is, strictly speaking, no longer true for finite core vortices as an additional length scale, namely, the vortex mean horizontal radius $R$, is introduced in the problem. This means that the same value of $D$ for two different values of $\gamma$ leads to two different values of $d / R$ and hence different values for the relative distance between the vortices.

The remaining of the paper focuses on the finite core equivalent to the discrete equilibria described by Eqs. (7)(10). A similar approach was used to determine families of finite core tripolar vortices in a previous study. ${ }^{18}$

\section{V-STATES AND THEIR LINEAR STABILITY}

We seek families of zigzag-shaped hetonic quartets spanned by the distance between their inner vortices $d$ and for four different values of $\gamma=0.3,0.5,0.7,1.0$. The vortices have uniform PV. We could not determine numerically full branches of solutions for $\gamma \geq 1.2$ due to numerical convergence limitations. Without loss of generality, the vortices have a mean horizontal radius of $R=1$ and hence an area of $A=\pi R^{2}=\pi$. Their PV is set to $q= \pm 2 \pi$, and therefore their surface integrated $\mathrm{PV}$ is $\pm \kappa= \pm q A= \pm 2 \pi^{2}$. Figure 2 summarises the global characteristics $(d, b, \omega)$ for the finite core rotating zigzag-shaped hetonic quartets, and Fig. 3 presents the characteristics $(d, b, V)$ for the finite core translating zigzag-shaped hetonic quartets. In comparison, the results for the discrete zigzag-shaped hetonic quartets with $K=2 \pi^{2}$ are shown in the same figures. We recover two asymptotes for $b=f(d)$ in both cases. We see that $b \rightarrow \infty$ as $d \rightarrow 0$ and $b \rightarrow d$ as $d \rightarrow \infty$. In other words, the limiting cases are (i) a single vortex spanning both layers at the centre of the domain $(d \rightarrow 0)$ with two opposite-signed vortices ejected to infinity $(b \rightarrow \infty)$, (ii) a cold heton and a hot heton $(b \sim d)$ infinitely distant $(d \rightarrow \infty)$ for the rotating quartets and (i) a central heton $(d \rightarrow 0)$ with two satellites of different signs at infinity $(b \rightarrow \infty)$, (ii) again, two cold hetons or two hot hetons $(d \sim b)$ infinitely distant $(d \rightarrow \infty)$ for the translating quartets. The results $b=f(d)$ between the finite core and the discrete solutions are in good agreement. This is expected for global quantities such as the vortex centroid locations.

The discrepancy between the results obtained for finite core vortex equilibria and discrete vortex equilibria is due to the facts that (a) the vortex charge $\kappa$ is less localised in the finite
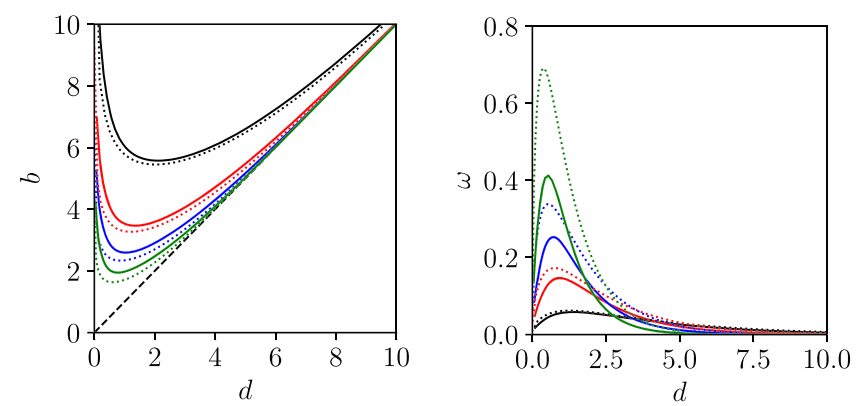

FIG. 2. Left: $b=f(d)$ for $\gamma=0.3$ (black), 0.5 (red), 0.7 (blue), 1 (green). Solid lines correspond to finite core vortices; dotted lines correspond to point vortices. The dashed line indicates the asymptote $b=d$. Right: Angular velocity $\Omega=g(d)$, same conventions. 

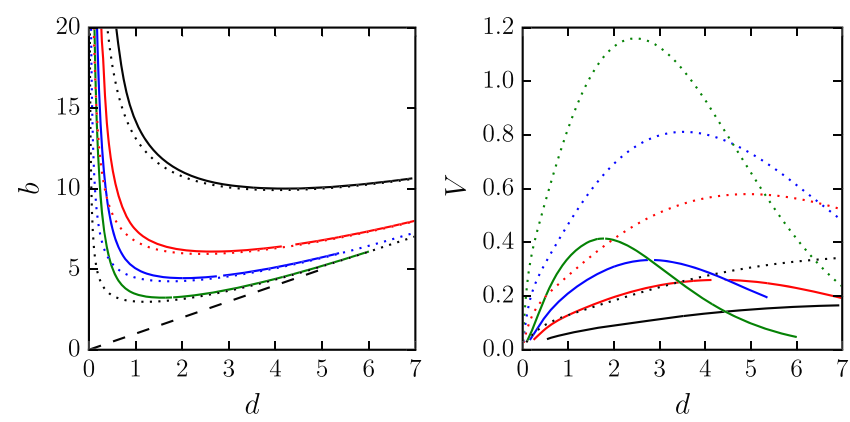

FIG. 3. Left: $b=f(d)$ for $\gamma=0.3$ (black), 0.5 (red), 0.7 (blue), 1 (green). Solid lines correspond to finite core vortices; dashed lines correspond to point vortices. The dot-dashed line indicates the asymptote $b=d$. Right: Translation velocity $V=l(d)$, same conventions.

core problem as the vortices spread over some horizontal distance (their radius $R$ ) and (b) in particular finite core vortices in different layers may horizontally overlap, while the discrete vortices for the same value of $(d, b)$ would still be clearly separated. This is in particular true for the inner vortices when $d$ is small. Recall that Eqs. (7) and (9) imply that the characteristics of the discrete equilibria collapse to single curves. However, as mentioned earlier, different values of $\gamma$ for identical values of $D, B$ imply different values for $d / R, b / R$ as $R=1$ for all $\gamma$. This is made evident by looking at the exact arrangement and shape of the finite core equilibria at the turning point $\left(d^{*}, b^{*}\right)$ of the graph $b=f(d)$, which corresponds to a single point of the graph $B$ vs $D$ for the discrete vortices. The shape of the vortices is shown in Fig. 4 for the finite core rotating zigzag-shaped hetonic quartets and in Fig. 5 for the finite core translating zigzag-shaped hetonic quartets. It is made clear that
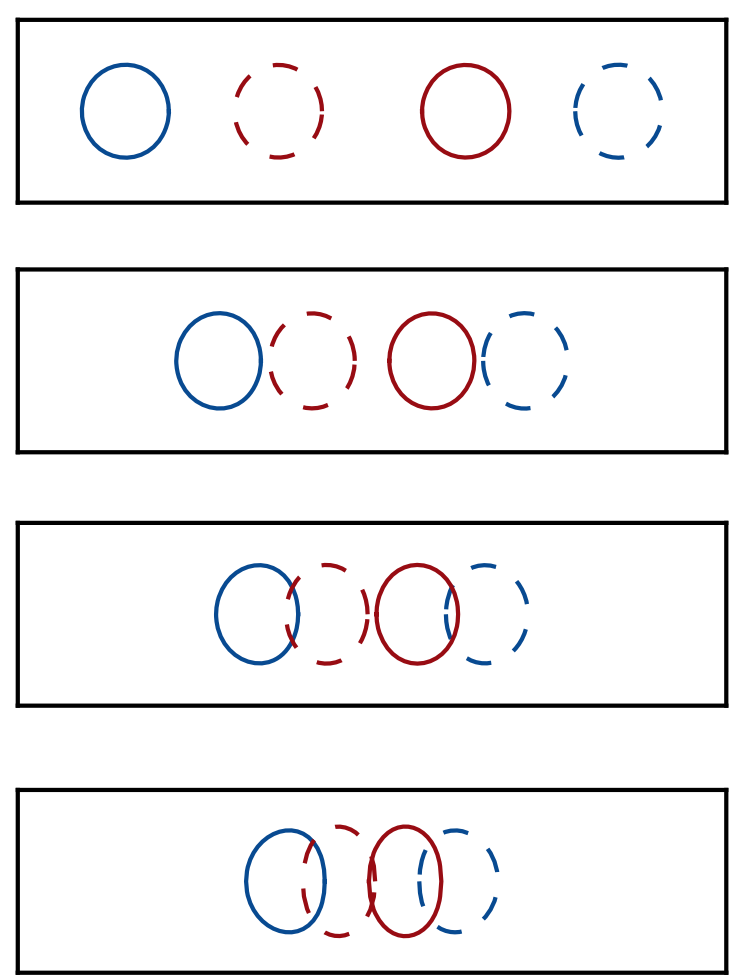

FIG. 4. Rotating hetonic quartets: shape of the finite-core vortices at the turning point in the curve $b=f(d)$ for $\gamma=0.3$ (first panel), 0.5 (second panel), 0.7 (third panel), and 1.0 (fourth panel) in a window $[-8,8] \times[-2,2]$.
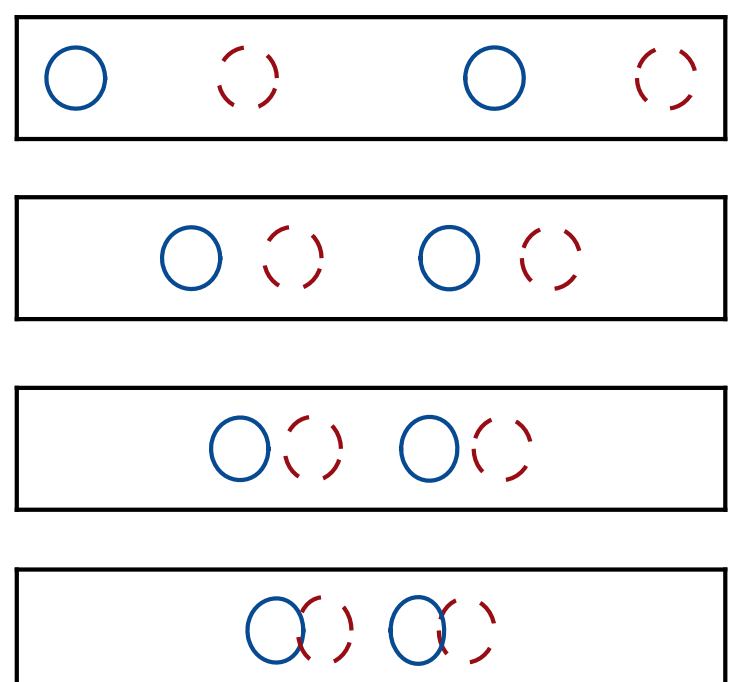

FIG. 5. Translating hetonic quartets: shape of the finite-core vortices at the turning point in the curve $b=f(d)$ for $\gamma=0.3$ (first panel), 0.5 (second panel), 0.7 (third panel), and 1.0 (fourth panel) in a window $[-12,12] \times[-2,2]$.

the relative distances $d / R, b / R$ are very different and hence that the characteristics of the equilibria cannot be expected to be the same. They cannot be exactly deduced from each other by a simple scaling by $\gamma$. When the vortices are well separated horizontally, their boundary is nearly circular as a result of the weak interactions. This is also the case when two vortices lying in different layers align near the centre $(d \rightarrow 0)$ or at infinity $(d \sim b \rightarrow \infty)$. This is due to the fact that isolated axisymmetric aligned hetons or aligned antihetons are in equilibrium. An antiheton is a pair of like-signed vortices lying in different layers. Only when the four vortices are close together, the shape of the vortices departs significantly from a circular cross section in response to both the horizontal shear and vertical shear the vortices induce on each other.

Figure 2 also shows the angular velocity $\omega$ of the rotating zigzag-shaped hetonic quartets. As expected, $\omega \rightarrow 0$ as $d \rightarrow 0$ and as $d \rightarrow \infty$. This is due to a combination of the compensation effect between the opposite sign vortices as the fact that $b \rightarrow \infty$ (in both cases). The results for the finite core rotating zigzag-shaped hetonic quartets and the discrete zigzag-shaped hetonic quartets are in qualitative agreement despite showing a significant difference in magnitude for $\omega$, in particular, for $d<2$. The difference in angular velocity $\omega$ can be attributed to the partial overlapping of the inner vortices in the finite core case. Results for the translation velocity $V$ of the translating zigzag-shaped hetonic quartets are presented in Fig. 3. Again, there is an overall qualitative agreement for the results obtained for the finite core vortices and the discrete vortices. The translation velocity vanishes as $d \rightarrow \infty$ and $d \rightarrow 0$ and reaches a maximum for a finite value of $d$ which depends on $\gamma$. Recall that Eq. (8) guarantees that the curves of $V$ collapse however to a single curve in variables $(D, B)$ for the discrete vortices. We again see a discrepancy in the magnitude of the velocity. The velocity of the discrete equilibria is higher than the velocity of the finite core equilibria; this is attributed to the fact that all the PV is concentrated on singular charges in the discrete approach rather than spread over finite areas. 
We next turn our attention to the linear stability of the zigzag-shaped hetonic quartets. The linear stability of the finite core quartets is examined by analyzing the eigenmodes (azimuthal normal modes) of deformation of the vortex bounding contours, as done in a previous study. ${ }^{18}$ The contour deformation azimuthal normal modes are proportional to $e^{\sigma t}$, where $\sigma=\sigma_{r}+\mathrm{i} \sigma_{i}$ is a complex number. The growth rates of a mode are therefore $\sigma_{r}$, while $\sigma_{i}$ is its frequency. One of the modes analyzed corresponds to a global displacement of the individual vortices. ${ }^{26} \mathrm{We}$ also analyze the linear stability of the discrete equilibria. To this purpose, we explicitly linearise the equations of motion of the discrete vortices about the equilibrium, with respect to infinitesimal perturbations on the vortex coordinates $\left(x_{i}, y_{i}\right)_{i=1,4}$. Again, the perturbations are taken as normal modes proportional to $e^{\sigma t}$. The terms of the linearised equations can be formally expressed by taking second order partial derivatives of Green's functions (6). The linearised system leads to a $8 \times 8$ algebraic eigenvalue problem which is solved numerically.

Linear stability results for the rotating zigzag-shaped hetonic quartets are presented in Fig. 6. Rotating zigzag-shaped hetonic quartets are (neutrally) stable for small values of $d$ as the largest growth rate is $\sigma_{r}=0$. An unstable mode appears for $d \geq d_{c}$, where $d_{c}$ is a threshold depending on $\gamma$ for both the discrete and finite-core vortices. We denote $d_{c}^{v}$ the stability threshold obtained for the discrete vortices and $d_{c}^{f}$ the stability threshold obtained for the finite-core quartets. The thresholds $d_{c}^{v}$ correspond in fact to a unique non-dimensional value $D_{c}^{v}=\gamma d_{c}^{v}$, while the growth rate of the modes scales as $\gamma^{2}$. The latter scaling comes from the scaling of the velocity gradients. The mode of instability is observed for both the discrete vortices and the finite core quartets, and the values of the thresholds $d_{c}^{v}$ and $d_{v}^{f}$ obtained for the two models are in remarkable agreement. We therefore deduce that the instability is physically related to a relative displacement of the vortices. The stability threshold $d_{c}^{f}$ for the finite core zigzagshaped hetonic quartets also scales as $\gamma^{-1}$, at least at leading order. Recall however that the vortices for the same value of $D=\gamma d$ but different values of $\gamma$ have different shapes;

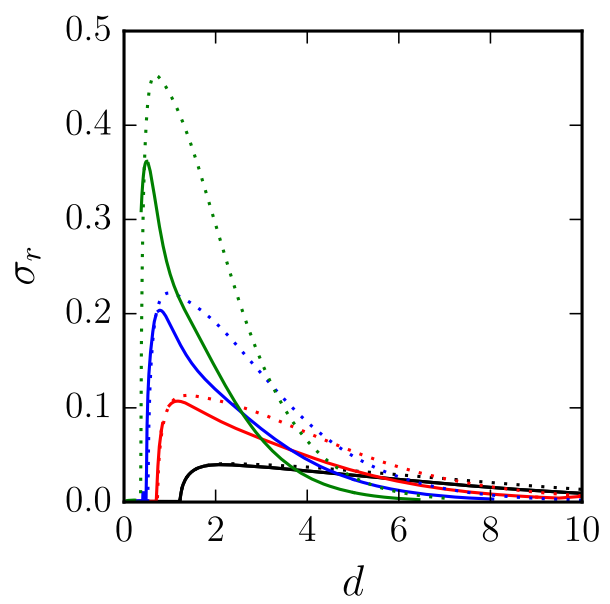

FIG. 6. Growth rate $\sigma$ of the most unstable mode for rotating hetonic quartets versus the distance $d$ for $\gamma=0.3$ (black), 0.5 (red), 0.7 (blue), 1 (green). Solid lines correspond to finite core vortices; dotted lines correspond to point vortices. see Fig. 4. As a consequence, one expects differences in the details of the instability for the finite-core zigzag-shaped hetonic quartets between cases with different $\gamma$. The growth rate of the instability reaches a maximum for a finite value of $d$ and slowly decreases back to zero as $d$ increases. The limit case of a central single vortex occupying both layers $(d \rightarrow 0)$ with two satellites ejected at infinity $(b \rightarrow \infty)$ is neutrally stable as one expects due to the lack of interaction. The discrepancy between the values of $\sigma_{r}$ is attributed to horizontal spreading and/or to overlapping of the finite-core vortices, as mentioned above. Finally, we note that a similar instability has been observed for three-dimensional quartets in a continuous stratification. ${ }^{32}$

The linear stability of the translating zigzag-shaped hetonic quartets is drastically different. The results are presented in Fig. 7. Using the same notations, the translating zigzagshaped hetonic quartets are all neutrally stable for $d>d_{c}^{v}$ (discrete quartets) or $d>d_{c}^{f}$ (finite core quartets), where $d_{c}^{v}$ is a lower stability threshold for the discrete translating zigzag-shaped hetonic quartets and $d_{c}^{f}$ is a lower stability threshold for the finite core translating zigzag-shaped hetonic quartets. These thresholds, again, correspond to a unique nondimensional value for the discrete translating zigzag-shaped hetonic quartets $D_{c}^{v}=\gamma d_{c}^{v}$. On the other hand, all translating zigzag-shaped hetonic quartets are unstable for $d<d_{c}$. The growth rate of the instability tends however to 0 as $d \rightarrow 0$. This is true for both the finite core and the discrete translating zigzag-shaped hetonic quartets, and the stability results are qualitatively similar for both models. Again, this means that the instability is related to a displacement of the vortices. The main difference between the two cases is that the range of the distance $d$ where the quartets are found unstable is narrower for the discrete vortices for the finite core vortices. The stability threshold $d_{c}^{v}$ for the discrete vortices, for a given $\gamma$, is also smaller than $d_{c}^{f}$ the stability threshold for finite core vortices. For the finite core translating zigzag-shaped hetonic quartets, the instability occurs typically in a region where $d / R<1$. The overlapping of the inner vortices is thus likely to strongly affect the instability. Results for the discrete vortices indicate that the

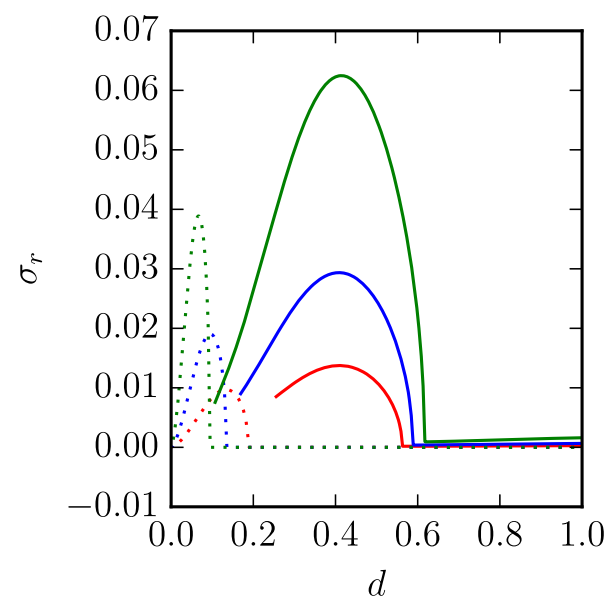

FIG. 7. Growth rate $\sigma$ of the most unstable mode for translating hetonic quartets versus the distance $d$ for 0.5 (red), 0.7 (blue), 1 (green). Solid lines correspond to finite core vortices; dashed lines correspond to point vortices. 
instability only occurs when some PV is found very close to the centre of the domain $\left(d<d_{c}^{v} \ll 1\right)$. For finite core vortices, some of the PV extends near the centre of the domain even for $d>d_{c}^{v}$, and this may trigger the instability.

\section{NONLINEAR EVOLUTIONS}

We next present the nonlinear evolution of the zigzagshaped hetonic quartets on four selected examples. In the first one, we consider the evolution of a finite core rotating zigzagshaped hetonic quartet for $\gamma=1, d=0.492>d_{c}^{f}, b=2.02$, and $\omega=0.419$. The results are presented in Fig. 8. The vortices are shown in a reference frame steadily rotating at the equilibrium angular velocity $\omega$; hence any motion observed in this frame corresponds to a departure from the equilibrium. No explicit perturbation is superimposed to the initial conditions. Small perturbations arise naturally from the numerical noise. The finite core rotating zigzag-shaped hetonic quartet remains in near equilibrium for a long period of time, up to $t \sim 25 \simeq 1.67 T_{r o t}$, where $T_{r o t}=2 \pi / \omega \simeq 15$ is the rotation period of the quartet. Then the four initially co-planar vortices start to drift apart and become misaligned. The vortices start to deform as a result of their relative displacement. It should be noted however that the vortices do not undergo strong deformations and that they remain compact patches of PV. They are not destroyed nor broken down into smaller vortices and filaments by the shear and strain they induce on each other. This is another indication that the instability primarily affects the location of the vortex centroids rather than their shape. Pure deformation modes often result in the breaking or merger of the vortices. ${ }^{25,33}$
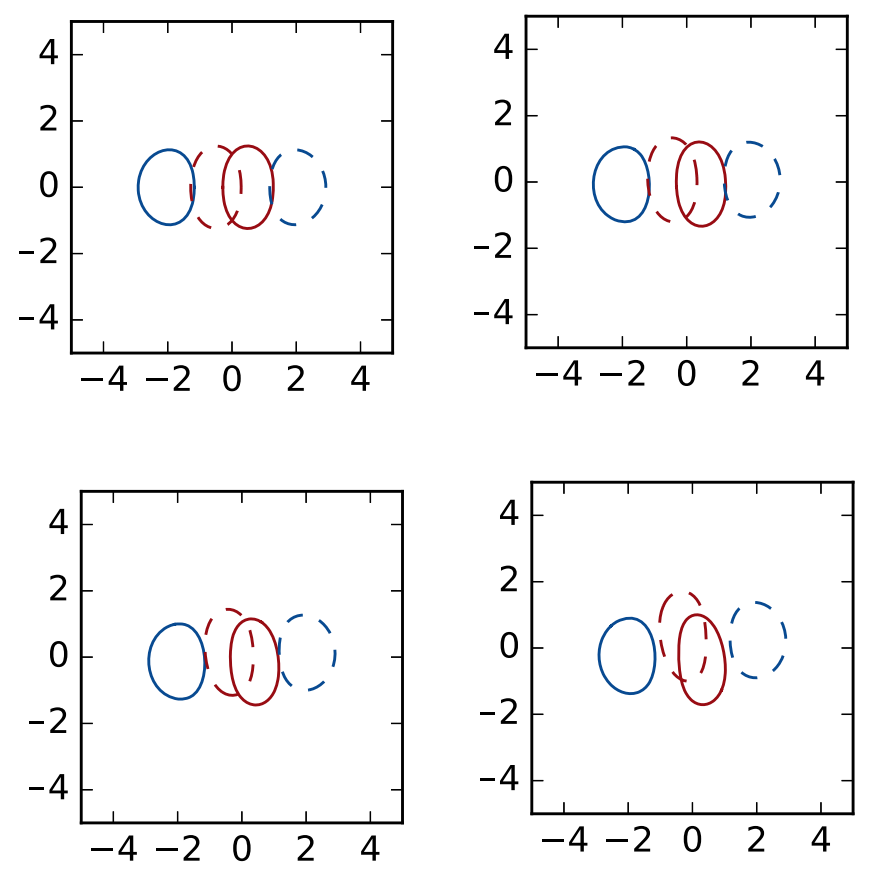

FIG. 8. Top view of the vortex bounding contours for the unstable rotating hetonic quartet with $\gamma=1, q= \pm 2 \pi, A=\pi, d=0.492, b=2.02, \omega=0.419$ at $t=0,26,28$, and 30 . Solid contours represent vortices in the top layer, while dashed contours represent vortices in the bottom layer. The views are shown in the reference frame rotating at the equilibrium angular velocity $\omega$.

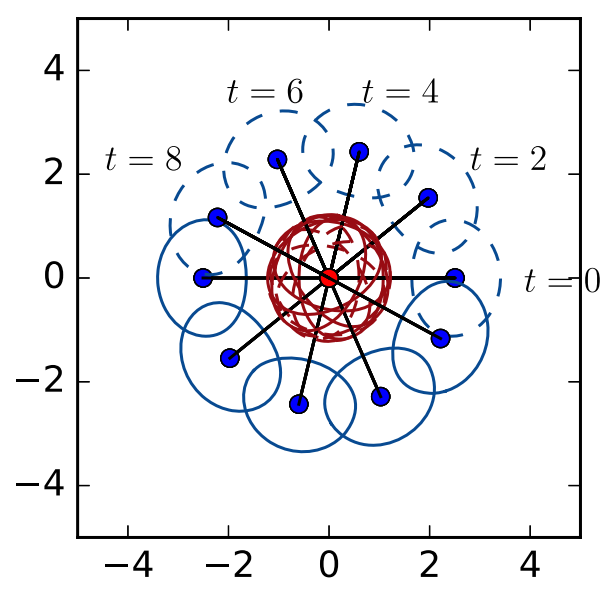

FIG. 9. Top view of the vortex bounding contours for the stable finite core rotating zigzag-shaped hetonic quartet with $\gamma=1, q= \pm 2 \pi, A=\pi, d=0.234$, $b=2.41, \omega=0.332$. Top view of the vortices at $t=0,2,4,6$, and 8 . Solid contours represent vortices in the top layer, while dashed contours represent vortices in the bottom layer. The views are shown in the fixed (non-rotating) frame to illustrate the steady rotation. The vortices remain in mutual equilibrium until the end of the simulation at $t=100$. The vortex centroids are indicated by small filled disks, and they are joined by a line to show the solid-state rotation.

The steady rotation of a stable finite core rotating zigzagshaped hetonic quartet is illustrated in Fig. 9. In this example, $\gamma=1, d=0.234<d_{c}^{f}, b=2.41$, and $\omega=0.332$. The vortices are shown in a fixed (non-rotating) frame to show the steady rotation of the quartet. The simulation was run till $t=100 \simeq 5.3 T_{\text {rot }}$, where $T_{r o t} \simeq 18.9$ is the rotation period of the quartet. The quartet was still steadily rotating at that time.

We next turn to finite core translating zigzag-shaped hetonic quartets. In the first example, $\gamma=1, d_{c}^{f}=0.416<d_{c}^{f}$, $b=0.5 .93, V=0.163$, and the quartet is unstable. Results are presented in Fig. 10 in a reference frame translating at $V$. Comparing the typical order of magnitude of the growth rates obtained for the rotating zigzag-shaped hetonic quartets and the translating zigzag-shaped hetonic quartets, we see that the instability affecting the latter is weaker than the instability
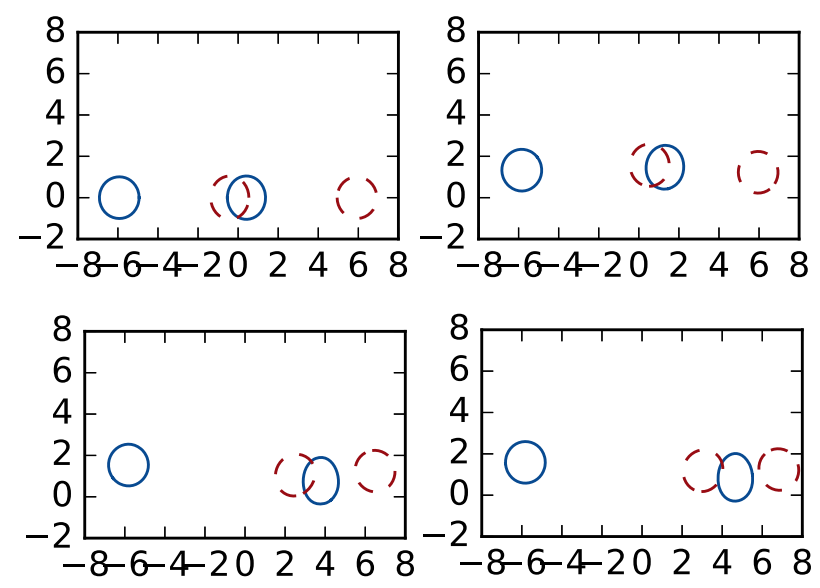

FIG. 10. Top view of the vortex bounding contours for the unstable finite core translating zigzag-shaped hetonic quartet with $\gamma=1, q= \pm 2 \pi, A=\pi$, $d=0.416, b=5.93, V=0.163$ at $t=0,400,425$, and 430. Solid contours represent vortices in the top layer, while dashed contours represent vortices in the bottom layer. The views are shown in the reference frame translating at the equilibrium velocity. 


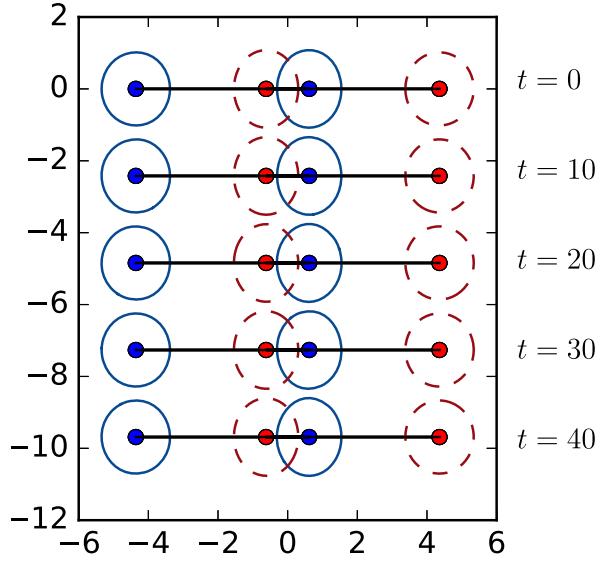

FIG. 11. Stable translating hetonic quartet for $\gamma=1, q= \pm 2 \pi, A=\pi$, $d=0.619, b=4.35, V=0.242$. Top view of the vortices at $t=0,10,20$, and 40 . Solid contours represent vortices in the top layer, while dashed contours represent vortices in the bottom layer. The views are shown in the fixed (non-rotating) frame to illustrate to steady rotation. The vortices remain in mutual equilibrium until the end of the simulation at $t=500$. The vortex centroids are indicated by small filled disks, and they are joined by a line to show the uniform translation.

affecting the former, with maximum growth rates almost an order of magnitude smaller. The instability typically grows at a slower pace for the translating zigzag-shaped hetonic quartets. Indeed, in the present example, the vortices in the quartet are only slightly deflected from their equilibrium position by $t=400 \simeq 12.4 T_{\text {trans }}$, where $T_{\text {trans }}=R / V$ is a translation time scale set to the time taken by the quartet to move forward by a vortex radius. The vortex configuration becomes asymmetric with the central heton moving to the right getting loser to the right-hand outermost vortex satellite. Again, we observe a small variation in the shape of the vortices due to their relative displacement (hence vortices are subjected to time dependent strain and shear). The interaction is nonetheless not destructive, and the four vortices conserve their initial volume. Such an exchange of vortex partner is similar to the heton evolutions observed in the ocean.

We finally illustrate the steady translation of a stable finite core translating zigzag-shaped hetonic quartets for $\gamma=1$, $d=0.619>d_{c}^{f}, b=4.35$, and $V=0.242$. Here $T_{\text {trans }} \simeq$ 4.13. The simulation was run up to $t=500 \simeq 120 T_{\text {trans }}$, where no sign of deviation was observed. Results are presented in Fig. 11 in a fixed (non-translating) reference frame, where the uniform translation of the quartet is made evident.

\section{CONCLUSION}

We have numerically determined two new families of equilibrium states (V-states) for finite core rotating and translating zigzag-shaped hetonic quartets. These states stem from the existence of equilibrium solutions for configurations of four co-planar discrete vortices. The families are characterised by the value of $\gamma$, which is the inverse Burger number, and are spanned by the distance $d$ separating the two innermost vortices of the quartets. We have found that rotating zigzagshaped hetonic quartets are unstable for $d>d_{c}$, where $d_{c}$ is a $\gamma$-dependent threshold. For discrete rotating, the value of $d_{c}^{v}$ corresponds to a unique non-dimensional of $D_{c}^{v}=\gamma d_{v}^{c}$.
This is also true at leading order for the finite core rotating zigzag-shaped hetonic quartets. The growth rate of the instability reaches a maximum for a finite value of $d$ and vanishes as $d \rightarrow \infty$. The situation is reversed for translating zigzag-shaped hetonic quartets. Indeed such quartets are neutrally stable for $d>d_{c}$, and only quartets with $d<d_{c}$ are found unstable. The instability is typically weaker than for the translating rotating zigzag-shaped hetonic quartets as the growth rate of the instability for the translating quartets is typically an order of magnitude smaller than the growth rate of the instability for the rotating quartets. The mode of instability is recovered (at least qualitatively) when modeling the quartets by discrete vortices. This confirms that the instability is related to a displacement of the vortex centroids.

We conclude that while rotating zigzag-shaped hetonic quartets are unstable for a large range of the parameter $d$, most of the translating zigzag-shaped hetonic quartets are neutrally stable as the instability only occurs for a small range $0<$ $d<d_{c}^{d}<1$, from our results. It should also be noted that the growth rates of the instability grow as $\gamma$ grows which is when the baroclinic effects increase (a two-dimensional barotropic equivalent flow is recovered for $\gamma \rightarrow 0$ ).

The new family of stationary states obtained in this paper supplements the class of known stationary solutions in a twolayer rotating fluid. ${ }^{18,34-39}$ Finally we note that rotating quartets exist in the ocean as an intermediate stage in the interaction of two hetons. ${ }^{3,4}$ This was observed in the Gulf of Cadiz in the eastern part of the Atlantic Ocean.

\section{ACKNOWLEDGMENTS}

J.N.R. is grateful to Professor David Dritschel for providing the two-layer contour surgery numerical code and for helpful discussions when designing the tools to obtain finite core steady states and to address their linear stability.

M.A.S. and X.C. were supported by RFBR/CNRS (PRC Grant No. 16-55-150001/1069). M.A.S. was also supported by RFBR (Grant No. 16-05-00121), RSF (Grant No. 14-5000095, geophysical applications), and MESRF (Grant No. 14.W.03.31.0006, numerical simulation, vortex dynamics).

${ }^{1}$ N. G. Hogg and H. M. Stommel, "The heton, an elementary interaction between discrete baroclinic geostrophic vortices, and its implications concerning eddy heat-flow," Proc. R. Soc. A 397, 1-20 (1985).

${ }^{2}$ Ring Group, "Gulf-stream cold-core rings: Their physics, chemistry and biology," Science 212, 1091-1100 (1981).

${ }^{3}$ X. Carton, N. Daniault, J. Alves, L. Chérubin, and I. Ambar, "Meddy dynamics and interaction with neighboring eddies southwest of Portugal: Observations and modeling," J. Geophys. Res. 115, 1-23, https://doi.org/10.1029/2009jc005646 (2010).

${ }^{4}$ P. L'Hegaret, X. Carton, I. Ambar, C. Menesguen, B. Hua, L. Chérubin, A. Aguiar, B. Le Cann, N. Daniault, and N. Serra, "Evidence of mediterranean water dipole collision in the gulf of cadiz," J. Geophys. Res. 119, 5337-5359, https://doi.org/10.1002/2014jc009972 (2014).

${ }^{5} \mathrm{~V}$. M. Gryanik, "Dynamics of singular geostrophic vortices in a two-level model of atmosphere (ocean)," Izvestiya, Atmos. Ocean. Phys. 19, 171-179 (1983).

${ }^{6} \mathrm{~V}$. M. Gryanik, "Dynamics of localized vortex perturbations "vortex charges' in a baroclinic fluid," Izvestiya, Atmos. Ocean. Phys. 19, 347-352 (1983).

${ }^{7}$ N. G. Hogg and H. M. Stommel, "Hetonic explosions: The breakup and spread of warm pools as explained by baroclinic point vortices," J. Atmos. Sci. 42, 1465-1476 (1985). 
${ }^{8}$ V. M. Gryanik, 'Localized vortices-'vortex charges' and 'vortex filaments' in a baroclinic differentially rotating fluid," Izvestiya Atmos. Ocean. Phys. 24, 919-926 (1988).

${ }^{9}$ W. R. Young, "Some interactions between small numbers of baroclinic, geostrophic vortices," Geophys. Astrophys. Fluid Dyn. 33, 35-61 (1985).

${ }^{10}$ M. A. Sokolovskiy and J. Verron, "Finite-core hetons: Stability and interactions," J. Fluid Mech. 423, 127-154 (2000).

${ }^{11}$ M. A. Sokolovskiy and J. Verron, "Four-vortex motion in the two layer approximation: Integrable case," Regular Chaotic Dyn. 5, 413-436 (2000).

${ }^{12}$ M. A. Sokolovskiy and J. Verron, "New stationary solutions to the problem of three vortices in a two-layer fluid," Dokl. Phys. 47, 233-237 (2002).

${ }^{13}$ M. I. Jamaloodeen and P. K. Newton, "Two-layer quasigeostrophic potential vorticity model,’ J. Math. Phys. 48, 065601 (2007).

${ }^{14}$ M. A. Sokolovskiy and X. Carton, "Baroclinic multipole formation from heton interaction," Fluid Dyn. Res. 42, 045501 (2010).

${ }^{15}$ M. A. Sokolovskiy and J. Verron, Dynamics of Vortex Structures in a Stratified Rotating Fluid, Atmospheric and Oceanographic Sciences Library (Springer, Switzerland, 2014), p. 47.

${ }^{16} \mathrm{Z}$. Kizner, "Stability and transitions of hetonic quartets and baroclinic modons," Phys. Fluids 18, 056601 (2006).

${ }^{17}$ G. K. Vallis, Atmospheric and Oceanic Fluid Dynamics: Fundamentals and Large-Scale Circulation (Cambridge University Press, Cambridge, 2006).

${ }^{18}$ J. N. Reinaud, M. Sokolovskiy, and X. Carton, "Geostrophic tripolar vortices in a two-layer fluid: Linear stability and nonlinear evolution of equilibria," Phys. Fluids 29, 036601 (2017).

${ }^{19}$ D. G. Dritschel, "Contour surgery: A topological reconnection scheme for extended integrations using contour dynamics," J. Comput. Phys. 77, 240-266 (1988).

${ }^{20}$ N. Zabusky, M. Hughues, and K. Roberts, "Contour dynamics for the Euler equations in two dimensions," J. Comput. Phys. 30, 96-106 (1979).

${ }^{21}$ V. F. Kozlov, "The method of contour dynamics in model problems of the ocean topographic cyclogenesis," Izvestiya, Atmos. Ocean. Phys. 19, 635-640 (1983).

${ }^{22}$ R. T. Pierrehumbert, "A family of steady, translating vortex pairs with distributed vorticity," J. Fluid Mech. 99, 129-144 (1980).

${ }^{23}$ D. G. Dritschel, "The stability and energetics of corotating uniform vortices," J. Fluid Mech. 157, 95-113 (1985).

${ }^{24}$ D. G. Dritschel, "A general theory for two-dimensional vortex interactions," J. Fluid Mech. 293, 269-303 (1995).
${ }^{25}$ J. N. Reinaud and D. G. Dritschel, "The merger of vertically offset quasigeostrophic vortices," J. Fluid Mech. 469, 287-315 (2002).

26 J. N. Reinaud, "On the stability of continuously stratified quasi-geostrophic hetons," Fluid Dyn. Res. 47, 035510 (2015).

${ }^{27}$ J. N. Reinaud and X. Carton, "Existence, stability and formation of baroclinic tripoles in quasi-geostrophic flows," J. Fluid Mech. 785, 1-30 (2015).

${ }^{28}$ P. Luzzatto-Fegiz and C. Williamson, "Determining the stability of steady two-dimensional flows through imperfect velocity-impulse diagrams," J. Fluid Mech. 706, 323-350 (2012).

${ }^{29}$ D. Dritschel, T. Hmidi, and C. Renault, "Imperfect bifurcation for the quasi-geostrophic shallow-water equations," Arch. Ration. Mech. An. (submitted).

${ }^{30}$ H. Płotka and D. G. Dritschel, "Quasi-geostrophic shallow-water vortexpatch equilibria and their stability," Geophys. Astrophys. Fluid Dyn. 106, 574-595 (2012).

${ }^{31}$ H. Płotka and D. G. Dritschel, "Quasi-geostrophic shallow-water doublyconnected vortex equilibria and their stability," J. Fluid Mech. 723, 40-68 (2013).

${ }^{32}$ J. N. Reinaud and X. Carton, "The interaction between two oppositely travelling, horizontally offset, antisymmetric quasi-geostrophic hetons," J. Fluid Mech. 794, 409-443 (2016).

${ }^{33}$ J. N. Reinaud and D. G. Dritschel, "Destructive interactions between two counter-rotating quasi-geostrophic vortices," J. Fluid Mech. 639, 195-211 (2009).

${ }^{34}$ L. M. Polvani, N. J. Zabusky, and G. R. Flierl, “Two-layer geostrophic vortex dynamics: 1. Upper-layer v-states and merger,” J. Fluid Mech. 205, 215-242 (1989).

${ }^{35}$ L. M. Polvani, "Two-layer geostrophic vortex dynamics. 2. Alignment and two-layer v-states," J. Fluid Mech. 225, 241-270 (1991).

${ }^{36}$ V. G. Makarov, M. A. Sokolovskiy, and Z. Kizner, "Doubly symmetric finite-core heton equilibria,” J. Fluid Mech. 708, 397-417 (2012).

${ }^{37}$ J. N. Reinaud and X. Carton, "The stability and non-linear evolution of quasi-geostrophic hetons," J. Fluid Mech. 636, 109-135 (2009).

${ }^{38}$ B. Shteinbuch-Fridman, V. Makarov, X. Carton, and Z. Kizner, "Two-layer geostrophic tripoles comprised by patches of uniform potential vorticity," Phys. Fluids 27, 036602 (2015).

${ }^{39}$ B. Shteinbuch-Fridman, V. Makarov, and Z. Kizner, "Transitions and oscillatory regimes in two-layer geostrophic hetons and tripoles," J. Fluid Mech. 810, 535-553 (2017). 